\title{
Preface (Vol 37, issue 5)
}

\author{
Nadia Magnenat-Thalmann ${ }^{1}$
}

Accepted: 15 April 2021 / Published online: 19 April 2021

(c) The Author(s), under exclusive licence to Springer-Verlag GmbH Germany, part of Springer Nature 2021

The Visual Computer is a popular journal that receives more than 1200 submissions a year. About $20 \%$ of the submissions is accepted for publication. In addition, the Visual Computer is hosting a few special issues, among them the Computer Graphics Conference (CGI) Special Issue. To accelerate the publication of the regular accepted papers published online, this issue contains 25 regular papers:

1. Yi-Fei Pu et al., titled: "Structure revealing of low-light images using wavelet transform based on fractionalorder denoising and multiscale decomposition"

2. Peizhong Liu et al., titled: "Robust adaptive learning with Siamese network architecture for visual tracking"

3. Brian Karr et al., titled: "Optical effects on HDR calibration via a multiple exposure noise-based workflow"

4. Hongbo Bi et al., titled: "C2Net: a complementary cosaliency detection network"

5. Wenhong Tian et al., titled: "Back-projection-based progressive growing generative adversarial network for single image super-resolution"

6. Lavdie Rada et al., titled: "Image-selective segmentation model for multi-regions within the object of interest with application to medical disease"

7. Ignacio Hernández-Bautista et al., titled: "Automatic filter coefficient calculation in lifting scheme wavelet transform for lossless image compression"

8. Canan Bahce et al., titled: "Compression of geometry videos by 3 D-SPECK wavelet coder"

9. Nam Kim et al., titled: "A survey on online learning for visual tracking"

10. Yujing Sun et al., titled: "Understanding deep face antispoofing: from the perspective of data"

11. Xiao-Jun $\mathrm{Wu}$ et al., titled: "Correlation tracking with implicitly extending search region"

12. Shaofan Wang et al., titled: "3D human body skeleton extraction from consecutive surfaces using a spatialtemporal consistency model"

Nadia Magnenat-Thalmann

thalmann@miralab.ch

1 MIRALab-CUI, University of Geneva, Battelle, Building A, 7, Route de Drize, 1227 Carouge, Geneva, Switzerland
13. Yongxia Zhang et al., titled: "Simple and fast image superpixels generation with color and boundary probability"

14. Chengying Gao, et al., titled: "Automatic 3D virtual fitting system based on skeleton driving"

15. Xueqin Zhang, et al., titled: "Online multi-object tracking with pedestrian re-identification and occlusion processing"

16. Guoqing $\mathrm{Hu}$ et al., titled: "FRNet: an end-to-end feature refinement neural network for medical image segmentation"

17. Di Qi, et al., titled: "Divided Voxels: an efficient algorithm for interactive cutting of deformable objects"

18. Seung-Tak Noh et al., titled: "Parametric fur from an image"

19. Taner Cevik et al., titled: "A high-discriminative facial recognition method based on shape and grey-level appearances using landmark-points"

20. Bhakti Sonawane et al., titled: "Review of automated emotion-based quantification of facial expression in Parkinson's patients"

21. Ming Li et al., titled: "A novel application framework for self-supporting topology optimization"

22. Mohammad Rahmanimanesh et al., titled: "Fuzzy local intensity clustering (FLIC) model for automatic medical image segmentation"

23. Rajkumari Devi et al., titled: "Discriminative object tracking with subspace representation"

24. Tian Bai et al., titled: "Recognizing art work image from natural type- A deep adaptive depiction fusion method"

25. Chunmeng Wang et al., titled: "Fast exposure fusion of detail enhancement for brightest and darkest regions"

Nadia Magnenat Thalmann.

Editor-in-Chief, the Visual Computer.

Publisher's Note Springer Nature remains neutral with regard to jurisdictional claims in published maps and institutional affiliations. 